RESEARCH ARTICLE

\title{
Frequency and Antimicrobial Resistance Pattern of Extensive-Drug Resistance Salmonella typhi Isolates
}

\author{
Ashraf Memon, Mubashir Ahmed, Khalid Iqbal \\ Kharadar General Hospital, Aga Khan Road, Kharadar, Karachi, Pakistan
}

\begin{abstract}
Objectives: Extensive drug resistance (XDR) typhoid fever, caused by resistance strain of Salmonella typhi, is an emerging public health concern in Pakistan. XDR typhoid fever severely limits the therapeutic options in outbreak cities of Pakistan. Antimicrobial resistance (AMR) surveillance has been carried out at study hospital in Karachi and here reporting the 15 months surveillance data.
\end{abstract}

Methods: A cross-sectional study was conducted on 969 Salmonella typhi isolates obtained from the blood culture samples of suspected typhoid fever cases at Kharadar General Hospital (KGH) from Jan 2018 to Mar 2019. Isolates were tested against 8 antimicrobial agents by quality controlled disk diffusion technique. Salmonella typhi Isolates resistance to first- line drugs; ampicillin, chloramphenicol, and co-trimoxazole referred as Multi-drug resistant (MDR), Whereas first-line, second-line drug (Ciprofloxacin) and third generation Cephalosporin (Ceftriaxone) resistance were considered as XDR.

Results: Of total 969 isolates, 777 (80\%) were MDR showed resistance to all three first-line antibiotics and 517 (53\%) were XDR resistant to five antibiotics (Ampicillin, Chloramphenicol, Co-trimoxazole, Ciprofloxacin and Ceftriaxone). Azithromycin and Imipenem were seen sensitive to all Salmonella typhi isolates (100\%) among tested antibiotics. More than half of study S. typhi isolates 513 (53\%) came from children under five years of age, among them 284 (55\%) were XDR.

Conclusions: A large proportion of S. typhi isolates showed XDR-ST strain. Only Azithromycin and Imipenem were sensitive against XDR-ST strain. Current limited antibiotic option poses a serious threat of complete antibiotic failure in future against rapidly changing strain of S. typhi. J Microbiol Infect Dis 2020; 10(2):68-74.

Keywords: Typhoid fever, Multi-drug resistance, Extensive-drug resistance, Salmonella typhi, Surveillance

\section{INTRODUCTION}

Typhoid and paratyphoid fever are caused by Salmonella enterica serovars Typhi (S. typhi) and Paratyphi (S. Paratyphi). Typhoid fever (TF) is considered as an important global public health problem and a major cause of death and disability particularly in developing countries. Globally, $14 \cdot 3$ million cases of typhoid fever and paratyphoid fever occurred in 2017 including 136,000 deaths [1]. South Asia reported the largest number of typhoid cases, 10.3 million, in 2017 which accounts for $72 \%$ of global burden in 2017 with the highest incidence rate of 549 cases per 100000 Population [1]. Typhoid fever is endemic in Pakistan with the incidence rate of
451.7 typhoid fever cases per 100,000 persons/year [2]. TF is also most common bacterial infection found in children in Pakistan, with incidence rates as high as 1000 cases per 100,000 child per years reported from Karachi [1].

An increasing trend of antimicrobial resistant pattern in S. typhi poses a serious threat of complete antibiotics failure in future, to the public health concern. Multi drug resistance (MDR) with reduce susceptibility to fluoroquinolone in S. typhi and S. Paratyphi has been reported since 2000 in several countries including Pakistan [3-11]. Sporadic occurrence of extensively drug resistance (XDR) stains of $S$.

Correspondence: Dr. Mubashir Ahmed, Research Coordinator, Kharadar General Hospital, Aga Khan Road, Kharadar, Karachi, Pakistan

Email: mubashir.ahmed71@gmail.com

Received: 03 October 2019 Accepted: 22 March 2020

Copyright (C JMID / Journal of Microbiology and Infectious Diseases 2020, All rights reserved 
typhi, was initially reported from Bangladesh and India [12-14]. A largest XDR outbreak was reported from Pakistan [15]. This XDR outbreak was started in November 2016 from district Hyderabad then spread to other districts of the Sindh province. So far till Mar 2019, total 9,815 typhoid fever cases have been reported to the provincial health authorities from different district of Sindh province [16]. Out of these reported typhoid fever cases, 6,480 (66\%) were XDR-TF. Mostly XDR-TF 4514 (69.7\%) cases were reported from Karachi, the provincial capital of Sindh, with the overall XDR typhoid attack rate of $22 / 100,000$ population (16].

Increasing number of typhoid fever cases and isolation of XDR strain of Salmonella typhi in Karachi and other area of Sindh province have been continuously reporting with a serious threat of complete antibiotics failure against the rapid genetic mutation stain of $S$. typhi. This is an emerging public health emergency and demands proper surveillance of antimicrobial resistance pattern of XDR strain of Salmonella typhi particularly in the outbreak city of Karachi. The aim of current study was to report the antimicrobial resistance surveillance data of $S$. typhi from a private tertiary care hospital.

\section{METHODS}

A retrospective observational study was conducted on 969 archived Salmonella typhi isolates obtained from the blood culture samples of suspected typhoid fever cases reported at Kharadar General Hospital (KGH) during the period from Jan 2018 to Mar 2019. Kharadar General Hospital Karachi, is located in the district south of Karachi which is comprises of Layari, Saddar and Kemari towns (Sub-district). Layari and Saddar towns are the most densely populated towns of Karachi. Study hospital mostly received patients from these two towns. Salmonella typhi isolates were tested on automated blood culture system (Bactec Dickinson, USA) against 8 antimicrobial agents by quality controlled disk diffusion technique based on Clinical and Laboratory Standards Institute (CLSI) guidelines [17]. The antibiotics (Oxoid Ltd. Basingstoke, UK) tested against $S$. typhi isolates were included Ampicillin $(10 \mu \mathrm{g})$, Chloramphenicol (30 $\mu \mathrm{g})$, Co-trimoxazole $(25 \mu \mathrm{g})$, Ceftriaxone $(30 \mu g)$, Cefixime $(5 \mu g)$, Ciprofloxacin (5 $\mu g)$, Azithromycin $(15 \mu \mathrm{g})$ and Imipenem $(10 \mu \mathrm{g})$. The Antimicrobial Susceptibility Testing (AST) cut-off values, provided by the CLSI guidelines, were used to interpret the AST results as sensitive, intermediate and resistant.
Multidrug-resistant typhoid fever (MDR-TF) is defined as typhoid fever caused by Salmonella enterica serovar Typhi strains ( $S$. typhi), which are resistant to the first-line recommended drugs for treatment such as ampicillin, chloramphenicol, and co-trimoxazole. [11,16,18]. Whereas Extensive Drug Resistance typhoid fever (XDR-TF) is caused by $S$. typhi strains resistance to first-line drugs, along with second-line drug (fluoroquinolone) and third generation Cephalosporin (Ceftriaxone) [16]. The study was approved by Ethics Review Committee (ERC) of KGH.

\section{Data Analysis}

Study data was entered on Microsoft Excel 2013. SPSS version 16 was used to analyse the data. Frequency and proportion were calculated for categorical variables (like gender/ MDR-TF/ XDRTF). Whereas mean and standard deviation were taken for continuous variables (example age), Chisquare test was used to see the correlation between dependent (MDR-TF/ XDR-TF) and independent variables and $P$ value $<0.05$ was considered statistically significant. Data was presented in tables and graph.

\section{RESULTS}

A total of 969 typhoid fever cases were identified by isolation of Salmonella typhi from blood culture of 7217 suspected typhoid fever cases reported at $\mathrm{KGH}$ during the study period of 15 months. Of total 969 typhoid cases, more than half of the cases 538 (55.5\%) were male. Mean age was $6.6 \pm 8.06$ years (Table 1 ).

Majority of typhoid fever cases 885/969 (91.3\%) were children less than 14 years of age. Among them, MDR-TF was 711 (80.3\%) and XDR-TF was $473(53.4 \%)$. Of total 885 pediatric typhoid fever cases, 513 (57.9\%) were children under five years of age and 87 (9.8\%) were less than two years of age. Of less than five years, MDRTF was reported 415 (46.9\%) and XDR-TF was $284(32.0 \%)$ respectively. Whereas under two years, MDR-TF were reported 70 (7.9\%), and XDR-TF cases were 52 (5.8\%). Of total 969 typhoid cases, majority of MDR-TF 345 (35.6\%) and XDR-TF 232 (23.9\%) were fallen in the age group of 2-5 years (Table 1 ).

Of total 969 typhoid fever cases, 777 (80.2\%) were MDR-TF cases, showed resistance to all three first-line antibiotics (Ampicillin, Chloramphenicol and Co-trimoxazole), and 517 $(53.3 \%)$ were XDR-TF cases, resistant to first 
line drugs, second line drugs (Ciprofloxacin) and the third generation Cephalosporin (Ceftriaxone). Among total 969 cases, only 38 $(4.1 \%)$ typhoid fever cases have shown sensitivity to all tested antibiotics. There was no statistically significant correlation found between MDR-TF/ XDR-TF with different age groups and with gender (Table 2).

Highest Salmonella typhi resistance were seen against Ampicillin 863 (89.1\%), followed by Cotrimoxazole 803 (82.9\%), Chloramphenicol 800 (82.6\%), Cefixime 709 (73.2\%), Ceftriaxone 699 (72.1\%), and Ciprofloxacin 686 (70.8\%). Only two antibiotics (Azithromycin and Imipenem) were seen sensitive to all Salmonella typhi cases (Table 3).

During the 15 months surveillance period, on average 65 typhoid fever cases were reported in a month with minimum $14(1.4 \%)$ cases in Jan 2018 and maximum 136 (14.0) cases in Jun 2018. Of total 969 typhoid fever cases in 15 months, nearly half of the of typhoid fever cases $462(47.6 \%)$ were seen during only four months period from May to Aug-2018. Among these 462 typhoid cases, MDR were $366(79.2 \%)$ and XDR were $209(45.2 \%)$ respectively (Figure 1).

\section{DISCUSSION}

Current study reported the contribution of Kharadar General Hospital in notifying ongoing outbreak of XDR typhoid fever cases caused by the resistance strains of $S$. typhi in Karachi, Pakistan. Study reported the 15 months surveillance data of antimicrobial resistance pattern of $S$. typhi against tested antibiotics at study hospital. In Karachi during the study period (Jan-2018-Mar 2019), total 4339 XDR-TF cases were reported from different hospitals to the Provincial Disease Surveillance and Outbreak Response Unit (PDSRU) in Sindh, Pakistan [16]. Among these notified XDR-TF cases, study hospital contributed 12\% (517 XDR-TF Cases) in the city XDR-TF notification. Mostly XDR-TF cases reported from the lower socio-economic neighborhood of study hospital from Lyari and Saddar towns (Sub-district) of district South, Karachi. This ongoing largest outbreak of XDR-TF was started from Hyderabad city of Sindh in November 2016 and then spread in different district of Sindh including Karachi. [15]. So far (Nov 2016-Mar 2019) 6480
XDR-TF cases have been reported from all district of Sindh among them 4514 (70\%) XDRTF cases were from Karachi [16]. Before this outbreak, a study from Aga Khan University Hospital (AKUH) reported the three years (20092011) clinical laboratory review data which revealed 2 cases of ceftriaxone-resistant $S$. typhi [11]. Another study from AKU reported only 4 cases of ceftriaxone-resistant $S$. typhi from Pakistan during the last 3 years (2012-2015) [19].

Typhoid fever infection occurs through oro-fecal route and spread through drinking contaminated water and/or food, poor hygiene (hand wash), and poor sanitation. The risk of typhoid fever is considered high in densely populated district south of Karachi because of unavailability of safe drinking water, poor disposal of waste water and mixing of sewerage water in drinking water, poor personal hygiene (poor practice of hand wash before eating and after defecation) practices in children, very low typhoid vaccination coverage and limited surveillance of typhoid fever in the area. AKUH has tested 13 drinking water samples from the household of typhoid fever cases located in Lyari town, Karachi, and reported the presence of high quantity of Coliforms and/or thermos-tolerant $\mathrm{E}$. coli in tested water samples. AKUH also reported the molecular analysis of 55 community water samples using Polymerase Chain Reaction (PCR), and detected S. typhi DNA in $12(21.8 \%)$ water samples [16].

Children are the most vulnerable population group among typhoid fever cases. Study reported more than $91 \%$ of salmonella isolates came from children $\leq 14$ years of age and $53 \%$ under five years of age. Among pediatric typhoid fever cases, nearly one-third (32\%) cases were XDR TF. Similar findings were reported by previous studies conducted in typhoid endemic areas, have shown around one-quarter to onethird of pediatric typhoid fever cases were under five years of age [20]. During 15 month's surveillance period, peak typhoid fever cases so did MDR-TF and XDR-TF cases, were seen during the summer period from May to Aug 2018 (Figure 1). These months are considered moon soon rainy season in Pakistan when drinking water contamination and diarrheal disease cases are increases. 
Table 1. Demographic characteristics of Salmonella typhi isolates, MDR-TF \& XDR-TF Cases at study hospital, Karachi $(\mathrm{N}=969)$.

\begin{tabular}{|c|c|c|c|}
\hline Characteristics & Salmonella typhi Isolates N (\%) & MDR-TF", N (\%) & XDR-TF", N (\%) \\
\hline \multicolumn{4}{|l|}{ Sex } \\
\hline Male & $538(55.5)$ & $433(44.7)$ & $285(29.4)$ \\
\hline Female & $431(44.5)$ & $344(35.5)$ & $232(23.9)$ \\
\hline Total & $969(100.0)$ & $777(80.2)$ & $517(53.3)$ \\
\hline \multicolumn{4}{|l|}{ Age } \\
\hline Mean Age with SD & $6.51 \pm 7.68$ & & \\
\hline$<2$ years & $87(9.0)$ & $70(7.2)$ & $52(5.4)$ \\
\hline $2-5$ years & $426(44.0)$ & $345(35.6)$ & $232(23.9)$ \\
\hline $6-14$ years & $372(38.4)$ & $296(30.5)$ & $189(19.5)$ \\
\hline$>14$ years & $84(8.7)$ & $66(6.8)$ & $44(4.5)$ \\
\hline Total & $969(100.0)$ & 777 (80.2) & $517(53.3)$ \\
\hline \multicolumn{4}{|l|}{ Quarter Period } \\
\hline Q1-2018 & $70(7.2)$ & $49(5.1)$ & $12(1.2)$ \\
\hline Q2-2018 & $279(28.8)$ & $216(22.3)$ & $76(7.8)$ \\
\hline Q3-2018 & $304(31.4)$ & $249(25.7)$ & $201(20.7)$ \\
\hline Q4-2018 & $170(17.5)$ & $140(14.4)$ & $118(12.2)$ \\
\hline Q1-2019 & $146(5.1)$ & $123(12.7)$ & $110(11.4)$ \\
\hline Total & $969(100.0)$ & 777 (80.2) & 517 (53.3) \\
\hline
\end{tabular}

${ }^{*}$ MDR-TF $=$ Multi-drug resistance-Typhoid Fever

** XDR-TF= Extensive-drug resistance-Typhoid Fever

Table 2. Age, gender \& quarter base distribution and correlation with MDR-TF \& XDR-TF cases ( $N=969)$.

\begin{tabular}{|c|c|c|c|c|}
\hline Characteristics & MDR-TF N (\%) & P-Value & XDR-TF' N (\%) & P-value \\
\hline \multicolumn{5}{|l|}{ Age groups } \\
\hline$<2$ years & $70(9.0)$ & & $52(10.1)$ & \\
\hline $2-5$ years & $345(44.4)$ & & $232(44.9)$ & \\
\hline $6-14$ years & $296(38.1)$ & $P=0.940$ & $189(36.6)$ & $P=0.449$ \\
\hline$>14$ years & $66(8.5)$ & & $44(8.5)$ & \\
\hline Total & 777 (100.0) & & $517(100.0)$ & \\
\hline \multicolumn{5}{|l|}{ Gender } \\
\hline Male & $433(55.7)$ & & $285(55.1)$ & \\
\hline Female & 344 (44.3) & $P=0.795$ & $232(44.9)$ & $P=0.791$ \\
\hline Total & 777 (100.0) & & $517(100.0)$ & \\
\hline \multicolumn{5}{|l|}{ Quarter Period } \\
\hline Q1-2018 & $49(6.3)$ & & $12(2.3)$ & \\
\hline Q2-2018 & $216(27.8)$ & & $76(14.7)$ & \\
\hline Q3-2018 & 249 (32.0) & $P=0.075$ & 201 (38.9) & $P<0.05$ \\
\hline Q4-2018 & $140(18.0)$ & & $118(22.8)$ & \\
\hline Q1-2019 & $123(15.9)$ & & $110(21.3)$ & \\
\hline Total & 777 (100\%) & & $517(100.0)$ & \\
\hline
\end{tabular}

${ }^{*}$ XDR-TF= Extensive-drug resistance-Typhoid Fever 
Table 3. Antibiotics sensitivity and resistance pattern among Salmonella typhi Isolates (N=969).

\begin{tabular}{lccc}
\hline Antibiotics & Sensitivity N (\%) & Intermediate N (\%) & Resistance N (\%) \\
\hline Ampicillin & $106(10.9)$ & $0(0.0)$ & $863(89.1)$ \\
Chloramphenicol & $169(17.4)$ & $0(0.0)$ & $800(82.6)$ \\
Co-trimoxazole & $166(17.1)$ & $0(0.0)$ & $803(82.9)$ \\
Ciprofloxacin & $249(25.7)$ & $34(3.5)$ & $686(70.8)$ \\
Cefixime & $259(26.7)$ & $1(0.1)$ & $709(73.2)$ \\
Ceftriaxone & $268(27.7)$ & $2(0.2)$ & $699(72.1$ \\
Azithromycin & $969(100.0)$ & $0(0.0)$ & $0(0.0)$ \\
Imipenem & $969(100.0)$ & $0(0.0)$ & $0(0.0)$ \\
\hline
\end{tabular}

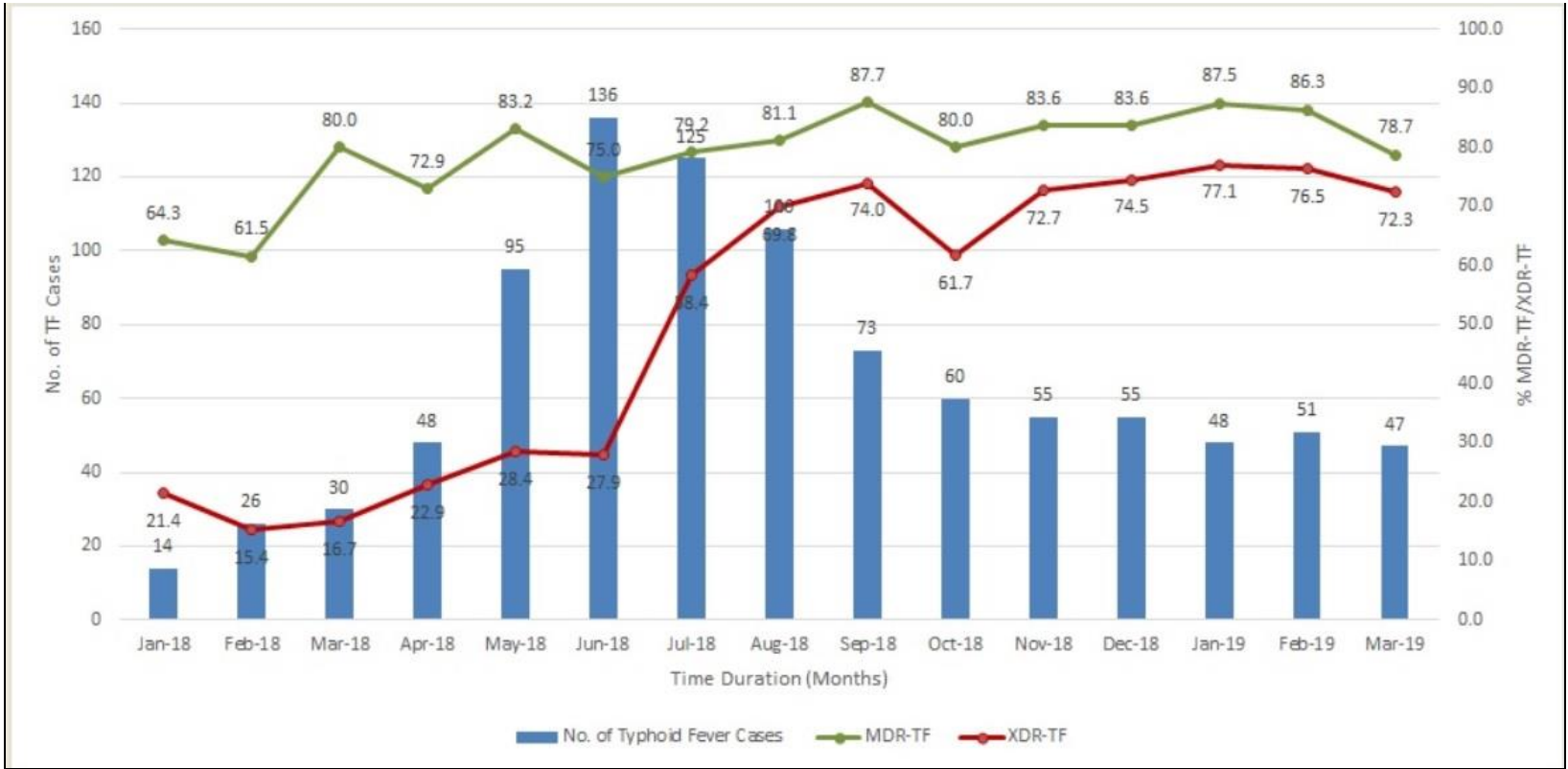

Figure 1. Showing monthly trend of Typhoid Fever, MDR-TF \& XDR-TF Cases over 15 months surveillance period at $\mathrm{KGH}$, Karachi (N=969)

Current study also revealed that XDR-TF proportion was initially low (17\%) in quarter 1 (Jan-Mar-2018) and $27 \%$ in quarter 2 (Apr-Jun2018) of surveillance period then exponentially rose to $66 \%$ in Quarter 3 (Jul-Sep-2018) and finally steady at $72 \%$ in Quarter 4 (Oct-Dec2018) and quarter 1 (Jan-Mar-2019) (Figure 1). This wide difference of rate of XDR-TF cases during surveillance period of Q1-2018 and Q12019 was due to rapid spread of genetic mutation strain of XDR-ST in the study catchment area of Lyari and Saddar towns of Karachi. Therefore, WHO recommend the antimicrobial resistance (AMR) pattern monitoring of Salmonella typhi in the outbreak area.
In a recent study, [21] the scientist has identified the genetic basis of a resistance strain of XDR typhoid that emerged in Pakistan. They conducted genome sequencing of XDR Salmonella typhi strains isolated from the outbreak region (Hyderabad and Karachi) of Sindh province of Pakistan. Genome sequencing revealed that all of the isolated belong to the H58 clade containing genes that impart resistance to chloramphenicol, ampicillin, and trimethoprim-sulfamethoxazole. Further analysis showed that they harbored a plasmid (IncY) that carries the blaCTX-M15 gene, which mediates resistance to ceftriaxone and qnrS fluoroquinolone resistance gene.

In Karachi only 10 hospitals have been reporting the typhoid fever cases to PDSRU without any 
formal reporting format. General practitioners (GPs) and private medical centers have received majority of typhoid fever cases from the community and there is no mechanism exist to report these cases to the concern health authorities. Also these GPs are treating patients presented with fever with broad spectrum antibiotics without any investigation. Furthermore antibiotics are easily available over the counter of medical stores without any physician prescription. Overuse or misuse of antibiotics without any rational can be a risk factor for emergence of XDR strain of S. typhi. Majority of physicians are still diagnosing the typhoid fever cases on clinical basis or through Widal test. They don't prescribe blood culture test either due to non-affordability of blood culture test cost by the patients or due to limited access of laboratories having antimicrobial susceptibility testing capacity, which ultimately limit the surveillance of XDR S. typhi.

Emergence of XDR stains of $S$. typhi, would severely limit the therapeutic option in Pakistan. Only available antibiotic options are Azithromycin and Carbapenem against XDR $S$. typhi. Cost effectiveness and only oral drug has made Azithromycin the current drug of choice for the treatment of XDR Typhoid fever in Pakistan. Carbapenem is expensive and give intravenously so use in admitted patients. Moreover expensive antibiotics option for XDRTF treatment would further raised the economic health burden of the country.

As the therapeutic options become more limited, further preventive measures should be urgently needed to take by the concern authority for the control of this prevail XDR-TF e outbreak in Sindh. These measures may include availability of safe drinking water, improve sanitation, mass vaccination campaign and health education to the affected communities regarding personal hygiene (hand washing before meal and after defecation), boiling drinking water, washing eatable fruits and vegetables, limit eating out particularly from street vendors. Also need to provide current updates on XDR-TF management to GPs and other typhoid treating physicians. At Kharadar general hospital during Sep 2018 to April 2019, nearly 10,000 children age 6 months to 10 years from the neighbourhood of Saddar and Lyari towns, have received the single dose of $\mathrm{WHO}$ prequalified
Typhoid Conjugate Vaccine (TCV) [1], having long-lasting immunity, with the support of AKU Project "Surveillance for Enteric Fever in Asia Project" (SEAP II).

Limitation: Re-confirmation of $S$. typhi resistance cases through minimum inhibitory concentration (MIC) of the antibiotics was not determined.

\section{Conclusion}

A large proportion of $S$. typhi isolates were XDRST with only sensitivity to Azithromycin and Imipenem antibiotics. Current limited treatment option would pose a serious threat in future for no choice of antimicrobial treatment option for the treatment of rapidly changing resistance strain of $S$. typhi. There should be a need of continuous surveillance of antimicrobial resistance pattern of $S$. typhi and enhance XDRST reporting network by including more hospitals and private practitioners in the outbreak areas.

\section{ACKNOWLEDGMENTS}

We would like to thanks the laboratory staff of Kharadar General Hospital, Karachi for data provision. We are also grateful to the administration of Kharadar General Hospital for their support for this surveillance survey. Data presenting here was solely collected and reported typhoid cases from KGH. There was no conflict of interest among authors. No funding was taken from any donors except Typhoid conjugate vaccine support from AKUH, Karachi.

Declaration of Conflicting Interests: The authors declare that they have no conflict of interest.

Financial Disclosure: No financial support was received.

\section{REFERENCES}

1. Stanaway JD, Reiner RC, Blacker BF, et al. The global burden of typhoid and paratyphoid fevers: a systematic analysis for the Global Burden of Disease Study 2017. Lancet Infect Dis 2019 Apr; 19(4):369-381.

2. Ochiai RL, Acosta CJ, Danovaro-Holliday MC, Baiqing D, Bhattacharya SK. A study of typhoid fever in five Asian countries: disease burden and implications for controls. Bull World Health Organ 2008; 86:260-268.

3. Azmatullah A, Qamar FN, Thaver D, Zaidi AK, Bhutta ZA. Systematic review of the global epidemiology, clinical and laboratory profile of enteric fever. J Glob Health 2015; 5(2):020407. 
4. Chau TT, Campbell JI, Galindo CM, et al. Antimicrobial drug resistance of Salmonella enterica serovar Typhi in Asia and molecular mechanism of reduced susceptibility to the fluoroquinolones. Anti Microb Agents Chemother 2007; 51:4315-23.

5. Parry CM. The treatment of multidrugresistant and nalidixic acid-resistant typhoid fever in Viet Nam. Trans R Soc Trop Med Hyg 2004; 7:413-422.

6. Hasan R, Cooke FJ, Nair S, Harish BN, Wain J. Typhoid and paratyphoid fever. Lancet 2005; 9497:1603-04.

7. Capoor MR, Nair D, Deb M, Aggarwal P. Enteric fever perspective in India: emergence of high-level ciprofloxacin resistance and rising MIC to cephalosporins. J Med Microbiol 2007; 8:113132.

8. Raveendran R, Wattal C, Sharma A, Oberoi JK, Prasad KJ, Datta S. High level ciprofloxacin resistance in Salmonella enterica isolated from blood. Indian J Med Microbiol 2008; 1:50-53.

9. Hasan R, Zafar A, Abbas Z, Mahraj V, Malik $F$, Zaidi A. Antibiotic resistance among Salmonella enterica serovars Typhi and Paratyphi A in Pakistan (2001-2006). J Infect Dev Ctries 2008; 4:289-294.

10. Mirza SH, Khan MA. Low -level quinolone resistance in multi-drug resistant typhoid. J Coll Physicians Surg Pak 2008; 1:13-16.

11. Qamar FN, Azmatullah A, Kazi AM, Khan E, Zaidi AK. A three-year review of antimicrobial resistance of Salmonella enterica serovars Typhi and Paratyphi A in Pakistan. J Infect Dev Ctries 2014; 8:981-986.

12. Saha SK, Talukder SY, Islam M, Saha S. A highly ceftriaxone-resistant Salmonella typhi in Bangladesh. Pediatr Infect Dis J 1999 Apr; 18(4):387.

13. Lakshmi V, Ashok R, Susmita J, Shailaja VV. Changing trends in the anti-biograms of Salmonella isolates at a tertiary care hospital in Hyderabad. Indian J Med Microbiol 2006; 1:45-48.

14. Bhattacharya SS, Das U, Choudhury BK. Occurrence \& antibiogram of Salmonella typhi \& S. Paratyphi A isolated from Rourkela, Orissa. Indian J Med Res 2011; 133:431-433.

15. Yousufzai MT, Qamar FN, Shakoor S, et al. Ceftriaxone-resistance Salmonella typhi Outbreak in Hyderabad City of Sindh, Pakistan: High Time for the Introduction of Typhoid Conjugate Vaccine. Clin Inf Dis 2019; 68(S1):S16-21.

16. Federal Disease Surveillance and Response Unit, National Institute of Health, Islamabad. Weekly field epidemiology report 2019 Mar 25-31; 2(13):1-8. Available: https://www.nih.org.pk/wp-
content/uploads/2019/04/FELTP-PakistanWeekly-Epidemiological-Report-March-25-312019.pdf.

17. Clinical and Laboratory Standards Institute. Performance Standards for Antimicrobial Susceptibility Testing; Twenty-Fifth Informational Supplement. CLSI document 2015 M100-S25.

18. Mutai WC, Muigai AWT, Waiyaki $P$, Kariuki S. Multi-drug resistant Salmonella enterica serovar Typhi isolates with reduced susceptibility to ciprofloxacin in Kenya. BMC Microbiology 2018; 18:187.

19. Qamar FN, Yousafzai MT, Sultana S, Baig A, Shakoor S, Hirani F et al. A retrospective study of laboratory-based enteric fever surveillance, Pakistan, 2012-2014. J Infect Dis. 2018 Nov 10;218(suppl 4):S201-S205. doi: 10.1093/ infdis/jiy205.

20. Lin $F Y$, Vo $A H$, Phan VB, et al. The epidemiology of typhoid fever in the Dong Thap Province, Mekong Delta region of Vietnam. Am J Trop Med Hyg 2000; 62: 644-648.

21. Klemm EJ, Shakoor S, Page AJ, et al. Emergence of an extensively drug-resistant Salmonella enterica serovar Typhi clone harboring a promiscuous plasmid encoding resistance to fluoroquinolones and third-generation cephalosporins. mBio 9.2018. doi:10.1128/mBio.00105-18. 